\title{
Circuit
}

Musiques contemporaines

\section{Introduction. Le « cas Dusapin »}

\section{Maxime McKinley}

Volume 29, numéro 1, 2019

Pascal Dusapin : la parallaxe des voix

URI : https://id.erudit.org/iderudit/1059423ar

DOI : https://doi.org/10.7202/1059423ar

Aller au sommaire du numéro

\section{Éditeur(s)}

Circuit, musiques contemporaines

ISSN

1183-1693 (imprimé)

1488-9692 (numérique)

Découvrir la revue

Citer ce document

McKinley, M. (2019). Introduction. Le " cas Dusapin ». Circuit, 29(1), 5-9.

https://doi.org/10.7202/1059423ar

Ce document est protégé par la loi sur le droit d'auteur. L'utilisation des services d'Érudit (y compris la reproduction) est assujettie à sa politique d'utilisation que vous pouvez consulter en ligne.

https://apropos.erudit.org/fr/usagers/politique-dutilisation/
Cet article est diffusé et préservé par Érudit.

Érudit est un consortium interuniversitaire sans but lucratif composé de l'Université de Montréal, l'Université Laval et l'Université du Québec à Montréal. Il a pour mission la promotion et la valorisation de la recherche. https://www.erudit.org/fr/ 


\section{Introduction. Le «cas Dusapin»}

Maxime McKinley

Beaucoup de forces opérantes agissent dans, par, sous et sur la musique de Pascal Dusapin ${ }^{1}$ et, plus généralement, à travers sa pensée et sa créativité. Investiguer cela est, déjà, une tâche inépuisable qui ne peut se faire sans convoquer au moins une part de curiosité universelle. Cela étant, en des termes plus circonscrits et situés, l'un des aspects de la pertinence de ce compositeur concerne le passage d'un siècle à l'autre. En ce sens, des comparaisons seraient sans doute possibles avec Alban Berg, un début de siècle plus tôt. Il s'agit là de deux configurations faites de transitions, de chevauchements, de chemins de traverse, entre deux temps et peut-être, parfois, hors du temps. En ce sens, le «cas Dusapin» peut sans doute être en partie perçu comme une illustration, en musique, de la déclaration décisive de la romancière Natacha Michel: «Le xxe siècle a eu lieu². » C'est-à-dire que l'on n'ignore pas ce qui s'y est passé, qu'on en prend acte, mais que l'on reconnaît aussi qu'on n'y est plus. Les «crises de l'art contemporain » relatives aux avant-gardes du $\mathrm{xx}^{\mathrm{e}}$ siècle deviennent ainsi caduques ou, à tout le moins, nécessitent d'être repositionnées. Ces mouvements artistiques représentent-ils une parenthèse fermée, un amas de tentatives à oublier et dont il faut sortir? Ou bien la pointe de l'iceberg d'un potentiel nouveau, au nom duquel il faut persévérer et insister, au risque d'une certaine monotonie académique contraire à ses aspirations initiales? Face à ces questions difficiles, l'œuvre de Dusapin m'évoque parfois l'image d'un navire passant de justesse entre deux récifs, ce passage n'étant rendu possible qu'au prix de ne pas rester rigide: cela demande des contorsions, des mouvements vifs, des déplacements de poids. Ce n'est pas un compromis tiède ou gris. C'est plutôt une aventure énergique, passionnée,

1. Pour un résumé du parcours de ce compositeur et d'autres informations utiles - relatives, notamment, à son catalogue -, on consultera la page qui lui est consacrée sur le site de Durand-Salabert-Eschig: https://www. durand-salabert-eschig.com/fr-FR/ Composers/D/Dusapin-Pascal.aspx (consulté le 14 janvier 2019).

2. Citée dans la dédicace d'Alain
Badiou (2005), Le Siècle, Paris, Seuil. incertaine, voire sauvage. 
3. Voir la collection complète: https:// revuecircuit.ca/collection/ (consulté le 14 décembre 2018). Mentionnons plus particulièrement le numéro "Espace Xenakis" (vol. 5, $\left.n^{\circ} 2,1994\right)$, puisque Dusapin - né, au jour près, 33 ans après le compositeur architecte (le 29 mai 1955) - fut son élève de 1974 à 1978.

4. À noter que la graphie de ce titre, alternant romain et italique, est souhaitée ainsi par le compositeur (tout comme il tient particulièrement à l'esperluette de Roméo \& Juliette).

5. Vol. 21, $n^{\circ} 2,2011$

6. Pascal Dusapin et Maxime McKinley (2017), Imaginer la composition musicale: correspondance et entretiens (2010-2016), Villeneuved'Ascq, Presses universitaires du Septentrion.

7. Leçon inaugurale prononcée le

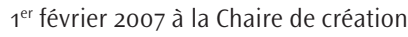
artistique du Collège de France; voir Pascal Dusapin (2009), Une musique en train de se faire, Paris, Seuil, p. 101-127.
C'est en cela notamment qu'il nous a semblé pertinent de consacrer un numéro de Circuit à Pascal Dusapin, renouant un instant avec les monographies de compositeurs d'envergure internationale, peut-être plus fréquentes dans les premières années de la revue 3 . Inutile toutefois de préciser combien Dusapin est le sujet de bien des sujets, et que dans cet univers foisonnant comme une toile de Riopelle, les mots «monographique» et «monothématique» sont des antonymes. Et si ce numéro a besoin d'une justification conjoncturelle plus spécifique, plus circonstanciée, alors on peut y placer comme point de fuite la création à venir, en septembre 2019 à la Monnaie de Bruxelles, du huitième opéra de Dusapin: Macbeth Underworld (2016-2018)4. Cet opéra, sur un livret de Frédéric Boyer d'après Shakespeare, sera ainsi créé trente ans après le premier opéra de Dusapin, Roméo Đ Juliette (19851988) - lui aussi en référence à Shakespeare, avec un livret d'Olivier Cadiot et créé en 1989. Comment trente années de créations lyriques irriguent-elles le travail de Dusapin, jusqu'à sa musique instrumentale, jusqu'aux arts visuels qu'il pratique? Voilà l'une des questions qu'il est possible de mettre en surplomb de ce numéro de Circuit. Mais en réalité, la mise en contexte de ce projet ne peut faire l'économie, plus prosaïquement, d'une tentative ratée qui, depuis, ne cesse de dévier en d'autres choses. Le $1^{\text {er }}$ septembre 2010, alors secrétaire de rédaction de Circuit, j’avais invité Dusapin à participer à une rubrique Enquête pour le numéro «Du spirituel dans l'art?5 », invitation qu’il déclina. Mais de ce refus est née une conversation épistolaire, elle-même suivie d'entretiens de vive voix, desquels un livre a découlé, paru en $2017^{6}$. Livre dans lequel nous discutons beaucoup des autres. Gli altri, pour faire allusion à ce personnage collectif (un sextuor vocal) de son opéra Passion (2006-2007). Peut-être parce que, selon le titre de sa leçon inaugurale au Collège de France, «Apprendre c'est devenir un autre ${ }^{7}$ ? Ou alors parce que, de nos jours, l'altérité est un enjeu sociétal et esthétique brûlant, comme l'était celui de la libération il y a quelques décennies? Quoi qu'il en soit, ce numéro de Circuit est - d’après des notions chères aux processus de création de Dusapin - à la fois une extension et un avers de ce livre. Ainsi, cette fois, ce sont plutôt les autres qui discutent de Dusapin. Du reste, certains de ces autres apparaissent aussi dans le livre...

Par hasard (ou, du moins, par une forme de connexité ne provenant pas d'une consigne extérieure), il est, dans chacun des textes de ce dossier, question de voix. Voix parlées, chantées, instrumentales, artistiques, gigognes... De surcroît, les voix de chacun des auteurs sont, par leurs contributions, comme autant de points de vue, ou de points d'écoute, sur les travaux de Pascal Dusapin, une voix en soi qui, un peu comme une figure fractale, 
fait elle-même appel à une panoplie de voix. C'est un véritable entrelacs ou rhizome de voix dont il s'agit là, chacune étant la ligne de fuite (expression) de choses captées (impression) $)^{8}$. Il y a la parallaxe stellaire des astronomes, la parallaxe de visée des photographes, la parallaxe en cinéma (permettant de marquer l'avant et l'arrière-plan), et bien d'autres encore'. Peut-être pourraiton imaginer, ici, une parallaxe des voix. La parallaxe est, essentiellement, l'incidence du déplacement d'un observateur $x$ sur l'observation d'un objet $y$. Une parallaxe des voix serait ainsi opérante dans des contextes où des voix (quelles que soient leurs natures) offrent des perspectives sur d'autres voix, comme des échos transformés et transformants de celles-ci. Cela implique entre elles des dynamiques d'affinités, de différences, d'indiscernabilités, de singularités. Cette réflexion vaut pour la musique considérée en immanence, mais aussi pour les abords extérieurs aux limites de celle-ci. Une sensibilité collective se dessine ainsi, par jeux de fuites et de captures, et c'est peutêtre ce qui fait dire à Dusapin, dans une formule particulièrement dense: «L'opéra c'est dire en chantant ce qui nous préoccupe ensemble ${ }^{10}$.»

On n'en finirait pas de déplier ce que cette définition de l'opéra selon Dusapin a de polysémique, notamment dans ses propres aventures opératiques. Mais remercions déjà Antoine Gindt de nous offrir de solides points de repère, par la traversée synoptique qu'il nous propose des sept premières créations lyriques de Dusapin. C'est ici le point de vue d'un producteur et metteur en scène qui a suivi cette aventure de très près, souvent de l'intérieur, depuis les débuts. En filigrane, on rencontre l'enjeu de la collaboration, des metteurs en scène aux librettistes, en passant par les chanteurs, chefs d'orchestre, et réalisateurs informatiques. La question même des délimitations de ce qu'est ou n'est pas l'opéra s'y pose, certaines œuvres pouvant avoir, à cet égard, un statut ambigu ${ }^{11}$. De là, avec l'article de Vincent Ranallo, nous passons en douceur du texte d'un metteur en scène à celui d'un chanteur. L'auteur s'y interroge sur ce qu'est « chanter Dusapin », en s'appuyant notamment sur des entretiens qu'il a menés avec deux des chanteurs parmi les plus significatifs dans le parcours du compositeur: la soprano Françoise Kubler et le baryton Georg Nigl. Ranallo a chanté la première nord-américaine d'O Mensch! (2008-2009) $)^{12}$, cycle qui fut créé par Nigl, et a ainsi vécu les tout débuts de la vie autonome de cette œuvre majeure. Or, on sait au moins depuis Jacques Amblard que la voix - parlée, chantée - est aussi au cœur de la musique instrumentale de Dusapin ${ }^{13}$. C'est ce qu'aborde Pierre Ouellet avec un point de vue de poète et d'essayiste, plus particulièrement autour de deux concertos récents de Dusapin: Aufgang (2011) et Outscape (2014) $)^{14}$. Lamour profond de Dusapin pour la littérature (en particulier Beckett) et la
8. Je pense ici à deux opus fondateurs de Dusapin: Musique captive (pour neuf instruments à vent) et Musique fugitive (pour trio à cordes), toutes deux de 1980.

9. À ce sujet, voir Slavoj Žižek, $L a$ parallaxe, Paris, Fayard, 2008.

10. Voir le texte de Dusapin sur Macbeth Underworld, dans la rubrique Documents de ce numéro.

11. À ce titre, par exemple, la musicologue Olga Garbuz a choisi de décrire comme septième opéra de Dusapin non pas Penthesilea (20112013), mais - prenant le titre au pied de la lettre - le spectacle sonore et pyrotechnique Opéra de feu, créé en 2010 par Dusapin (assisté de Thierry Coduys) et les artificiers du Groupe F (voir Olga Garbuz [2017], Pascal Dusapin: mythe, algorithme, palimpseste, Château-Gontier, Ædam Musicæ, p. 190 et p. 249).

12. À Montréal, le 7 février 2014 , dans le cadre de ma résidence (20112014) à la Chapelle historique du Bon-Pasteur, en collaboration avec la Société de musique contemporaine du Québec (SMCQ). Matthieu Fortin était au piano.

13. Jacques Amblard (2002), Pascal Dusapin, l'intonation ou le secret, Paris, Musica Falsa (réédité en 2018, chez le même éditeur, sous le titre Pascal Dusapin, le second style ou l'intonation).

14. Ces concertos sont les deux premiers volets d'un triptyque complété par At Swim-Two-Birds (20152016), et dont les instruments solistes sont respectivement le violon, le violoncelle, puis ces deux instruments ensemble. 
15. D'ailleurs, dans la foulée du "cas Wagner", Philippe LacoueLabarthe et, à sa suite, Alain Badiou nous ont bien montré combien les poètes et les philosophes peuvent avoir des intuitions puissantes sur les compositeurs: Philippe LacoueLabarthe (1991), Musica Ficta (figures de Wagner), Paris, Bourgois; Alain Badiou (2010), Cing leçons sur le "cas" Wagner, Caen, Nous.

16. À propos des "interlocuteurs imaginaires" de Dusapin (Beckett, Deleuze, Mandelbrot, Thom et bien d'autres), voir notamment Dusapin, 2009, op. cit.

17. À propos de ce verbe, Dusapin dit: "J'utilise souvent ce mot très ancien - pensez à ma pièce pour orchestre Extenso. J'aime ce mot parce qu'il indique l'idée de tendre vers l'eXtérieur, qui me semble plus fort qu'étendre, qui veut simplement dire allonger." (Dusapin, 2009, op. cit., p. 65.) philosophie (en particulier Deleuze) sont connus depuis longtemps, car le compositeur y recourt constamment comme points d'appui. Il nous paraissait donc naturel de convoquer un esthéticien et poéticien tel que Ouellet, afin d'appliquer un renversement à cette curiosité transdisciplinaire ${ }^{15}$. Nous passons ainsi, avec ces trois articles de fond de Gindt, Ranallo et Ouellet, des « extramusicalités» de l'opéra aux «intramusicalités» du concerto, a priori deux genres anciens. Et pourtant, on constate avec étonnement, à la lecture de ces textes - et a fortiori à l'écoute des œuvres dont il y est question -, combien tout cela fonctionne aussi à l'envers, avec la modernité de ces genres, les extramusicalités des concertos et les intramusicalités des opéras. Encore une fois, il faut trouver, pour appréhender Dusapin, des obliques: risquons ici les mots (à développer peut-être un jour) «paramusical» et «transmoderne».

Lire les écrits ou les entretiens de Dusapin, c'est comprendre que cet homme est traversé de flux et d'intensités. Cela peut venir de la nature, des moyens de comprendre celle-ci (les fractales de Mandelbrot et la morphogenèse de Thom, particulièrement $\left.{ }^{16}\right)$, mais aussi de la culture, ou plus simplement: des autres. Or, la rubrique Enquête de ce numéro, préparée par Emanuelle Majeau-Bettez, démontre que cela fonctionne aussi, là encore, dans l'autre sens. Par les témoignages recueillis de Barbara Hannigan, Samy Moussa, Armand Angster, Julie-Anne Derome et Julien Bilodeau, on découvre à quoi peuvent ressembler des rencontres - au sens fort - avec Dusapin. Et combien celles-ci ont des influences tangibles, fertiles, sur des vies et des voix musicales. Généreusement, ces participants ont confié dans leurs propos - par écrit dans un cas, viva voce pour les autres - comment et combien ils ont été traversés, chacun à leur manière, par un « effet Dusapin ».

Suit la rubrique Documents qui, à bien des égards, ex-tend ${ }^{17}$ cette Enquête. Si les compositeurs Moussa et Bilodeau doivent beaucoup à Dusapin (à l'instar du regretté Christophe Bertrand), il en est de même pour Igor Ballereau. Le premier document est un extrait d'un livre inédit de ce dernier, qui a commencé à composer après avoir rencontré Dusapin. Nous n'avons pas choisi cet extrait au hasard. Outre qu'il fait d'intéressantes observations musicales (notamment avec le concept de «voix gigognes»), il concerne le concerto pour trombone Watt (1994), œuvre emblématique de l'amitié créative de Dusapin avec le remarquable tromboniste québécois Alain Trudel. Le second document consiste en une lettre de la soprano canadienne Barbara Hannigan à Pascal Dusapin, rédigée lors de répétitions de l'opéra Passion. Le mélange de poésie et de professionnalisme, de fiction et de réalité y est très touchant. La reproduction de cette lettre fait aussi écho au témoignage de Hannigan dans l'Enquête. Enfin, le dernier document - et dernier texte de ce 
dossier thématique - a été écrit par Pascal Dusapin lui-même. Il s'agit d'un texte dense, sensible et grave sur son huitième opéra, Macbeth Underworld. Dusapin m’a fait parvenir ce texte le jour même où il déposa le manuscrit de son opéra chez son éditeur. C'est ainsi que ce Macbeth Underworld agit comme point de fuite à cette monographie. Mais ce n'est pas que par ce texte que Dusapin «se retourne»: on le voit un peu «de face » aussi grâce aux illustrations de ce numéro, entièrement de lui qui pratique, depuis si longtemps, le dessin et la photographie ${ }^{18}$. En bientôt 30 années d'existence et plus de 70 numéros, c'est la première fois que Circuit publie un dossier monographique dont le portfolio est constitué d'œuvres visuelles à part entière signées par le compositeur abordé19.

En complément à ce dossier monographique, on retrouve la rubrique Actualités. Si cette rubrique est généralement thématiquement indépendante, on remarquera tout de même quelques «agrafes». Ainsi, Marie Tachon évoque la création, en mai 2018, de l'opéra Lessons in Love and Violence de George Benjamin, à la Royal Opera House de Londres. Or, George Benjamin est sans doute, avec Wolfgang Rihm, l'un des compositeurs de sa génération dont Dusapin est le plus proche. Ce compte rendu est, en outre, l'occasion de recroiser Barbara Hannigan, aussi collaboratrice privilégiée de Benjamin. Ensuite, Marc Hyland souligne les vingt ans du Trio Fibonacci, dont la violoniste Julie-Anne Derome relate, dans l'Enquête, la magnifique rencontre avec Dusapin au Domaine Forget, en 2002. Enfin, Sharon Kanach nous convie à une traversée des écrits de François-Bernard Mâche, dans la foulée de deux parutions récentes. Cela nous permet de retrouver au passage Armand Angster et Françoise Kubler, interprètes emblématiques de Dusapin depuis ses débuts, puisqu'un concert de leur ensemble Accroche Note figure sur le DVD accompagnant l'un de ces ouvrages ${ }^{20}$. Ainsi, les branchements sont ici, comme souvent chez Dusapin, rhizomatiques.

Je profite de cette introduction pour souhaiter la bienvenue, sur notre comité de rédaction, à la musicologue Marie-Hélène Benoit-Otis, professeure à l'Université de Montréal. Et je tiens, en terminant, à remercier tous les participants de ce numéro, et tout particulièrement Pascal Dusapin et Patricia Alia $^{21}$, pour leur précieuse collaboration et leur amitié.

Bonne lecture!

Montréal, décembre 2018.
18. Voir, par exemple, Pascal Dusapin (2012), Accords photographiques, Paris, Éditions de la librairie de la galerie. Mentionnons aussi l'installation sonore et visuelle Mille Plateaux (2014), en collaboration avec Thierry Coduys pour la réalisation informatique (voir: https://www.milleplateaux.fr/ [consulté le 14 décembre 2018]).

19. Les antécédents les plus proches sont les extraits de partitions de quatuors à cordes de R. Murray Schaefer (vol. 11, $\mathrm{n}^{\circ}$ 12, 2000) - qui partage avec Dusapin d'avoir beaucoup composé pour cette formation - et les collages de cartes postales de Michel Butor et Henri Pousseur (vol. 12, $\mathrm{n}^{\circ} 1$, 2001).

20. Márta Grabócz et Geneviève Mathon (dir.) (2018), François-Bernard Mâche: le compositeur et le savant face à l'univers sonore, Paris, Hermann.

21. Patricia Alia est directrice des éditions Durand-Salabert-Eschig (Universal Music Publishing France). Je remercie également Enzo lannuzzi, assistant promotion aux mêmes éditions. 